\title{
Caractérisation des méthodes de coupe de bois dans les zones aménagée et non aménagée en Haute Casamance, Sénégal
}

\author{
Tamsir MBAYE ${ }^{1 *}$, Mamadou Ousseyni LY ${ }^{1}$, Maguette KAIRE ${ }^{2}$ et Paul NDIAYE ${ }^{3}$ \\ ${ }^{l}$ Centre National de Recherches Forestières (CNRF/ISRA), BP 2312 Hann Dakar, Sénégal. \\ ${ }^{2}$ Projet GCCA/CILSS Centre Régional AGRHYMET, Sénégal. \\ ${ }^{3}$ Université Cheikh Anta DIOP, Département de Géographie, BP 5005 Fann Dakar, Sénégal. \\ *Auteur correspondant, E-mail : tamsirmbaye76@gmail.com
}

\section{RESUME}

Cette étude aborde la problématique de l'exploitation des ressources ligneuses pour la production de charbon au Sud du Sénégal. Les différentes méthodes d'exploitation utilisées par zone aménagée (ZA) et non aménagée (ZNA) sont caractérisées. Dans chaque zone, six (6) placettes circulaires de $20 \mathrm{~m}$ de diamètre ont été délimitées pour l'inventaire des individus coupés. Les paramètres mesurés sont les classes de diamètre et hauteur de coupe, d'une part et les rejets de souches par catégorie de classe et de hauteur de coupe, d'autre part. Les résultats ont montré que les coupes ont concerné, dans la ZA, les classes de diamètre $[0,3[$ et $[3,6[$ avec respectivement $34,93 \%$ et $47,72 \%$, dans la ZNA, [0,3 [ avec 58,16\% et la hauteur de coupe au ras du sol avec $80,36 \%$ dans la ZA et $69,74 \%$ dans la ZNA. Pour la hauteur de rejets, celle moyenne, avec respectivement $54,02 \%$ et $49,44 \%$, et celle basse, avec respectivement $45,98 \%$ et $48,16 \%$, dominent dans la ZA et dans la ZNA. Ces résultats permettront une meilleure compréhension des effets des méthodes de coupe du bois sur la régénération par rejet de souche dans le but de mettre au point un plan de gestion durable des ressources ligneuses des formations forestières de la Haute Casamance basé sur la connaissance de la dynamique de régénération des espèces après coupe de bois.

(C) 2014 International Formulae Group. All rights reserved.

Mots clés: Sénégal, aménagement, hauteur de coupe, diamètre de coupe, régénération végétative.

\section{INTRODUCTION}

Les espaces forestiers du Sénégal font de plus en plus l'objet d'une exploitation accrue pour la production de bois d'énergie. En effet, considéré comme un pays boisé jusque dans les années 50 avec des savanes arbustives et arborées au nord (Louga, Saint Louis et Matam) et au centre (Kaolack, Fatick, Kaffrine) et des forêts au Sud (Casamance), le Sénégal voit son couvert végétal régresser d'année en année de 40000 ha (Boye, 2000 ;
Diagne, 2001). Cette situation est exacerbée par l'exploitation des zones forestières pour répondre à la forte demande en bois et/ou en charbon de bois des ménages urbains sénégalais (Boye, 2000 ; Sow et al., 2002; Faye et al., 2003) .

La réponse positive à cette forte demande en bois d'énergie renvoie aux capacités des espaces forestiers et/ou arborés exploités et, corollairement, aux modalités de coupe (hauteur et diamètre, saisonnalité, 
période, durée de rotation, seuils d'exploitation, ...) et à la capacité de régénération des arbres coupés. Il s'agit de multiples questionnements sur les processus naturels de régénération et les conditions d'aménagement durable des forêts des zones sèches assez mal connus (Bailly et al., 1982 ; Bellefontaine, 1997 ; Ribot, 1999).

Dans la zone aménagée, les prescriptions techniques du plan d'aménagement stipulent que les coupes concernent uniquement Combretum glutinosum et Terminalia macroptera, les diamètres de coupe sont compris entre 10 et $25 \mathrm{~cm}$ et les hauteurs de coupe au ras du sol. Dans la zone non aménagée, il n'y a pas d'indication sur les méthodes de coupe. Seul le quota (nombre de quintaux et zone) est fourni annuellement aux exploitants forestiers bénéficiaires par un décret du Ministre en charge de l'Environnement.

Peu d'études ont été effectuées au Sénégal sur la répartition des coupes pour la production de bois suivant la structure horizontale et verticale de la végétation ligneuse. Ainsi, notre travail a pour objectif de caractériser les pressions de coupe suivant la structure de la végétation et leurs effets sur la régénération dans les zones aménagée et non aménagée.

\section{MATERIEL ET METHODES Site d'étude}

Cette étude a été menée dans la zone soudanienne du Sénégal, précisément dans le département de Kolda, au niveau des communautés rurales de Saré Bidji et Ndorna (Figure 1), situées entre les isohyètes 1100 à $1200 \mathrm{~mm}$. Le cadre spatial a été la forêt communautaire de Saré Gardi ou zone aménagée (ZA) et les jachères de Coulabaly et Sinthian Djidéré ou zones non aménagées (ZNA) situées à $30 \mathrm{~km}$ de la zone aménagée (Figure 2). Les coupes dans la ZNA sont destinées à l'exploitation traditionnelle non réglementée contrairement à la ZA où les prescriptions techniques $\mathrm{du}$ plan d'aménagement doivent être suivies.

\section{Dispositif expérimental}

L'étude de la régénération des espèces ligneuses abattues coupées a été faite en délimitant 6 placettes circulaires de $20 \mathrm{~m}$ de diamètre dans la zone aménagée (ZA) et 6 autres dans la zone non aménagée (ZNA), soit 12 placettes distantes d'au moins $100 \mathrm{~m}$. L'emplacement et les distances entre les placettes ont été fortement influencés par le positionnement des anciens chantiers où la coupe de bois a été effectuée 12 mois plus tôt dans les ZA et ZNA. Les méthodes de coupe ont concerné la classe de diamètre des souches à couper et la hauteur de coupe. Six classes de diamètres [0,3 cm[, [3, $6 \mathrm{~cm}[,[6,9 \mathrm{~cm}[$, [9, $12 \mathrm{~cm}[$, [12, $15 \mathrm{~cm}$ [ et [15 $\mathrm{cm}$ et plus[ et cinq classes de hauteurs de coupe [ras du sol], [1, $10 \mathrm{~cm}[,[10,20 \mathrm{~cm}[,[20,30 \mathrm{~cm}$ [ et [30 cm et plus[ ont été étudiées dans les ZA et ZNA. Les rejets ont été classés en trois classes: basse, moyenne et haute.

L'inventaire a concerné toutes les souches des individus coupés. Les paramètres suivis sont le diamètre de la souche, la hauteur de coupe, le nombre de rejets, la hauteur des rejets le nombre de tiges. La méthode synchronique a été privilégiée pour disposer plus rapidement de données sur le terrain (Lepart et Escarre, 1984 ; MITJA, 1989 cités par Kairé, 2000).

\section{Analyse statistique}

Les données obtenues ont été soumises à des analyses de variances et les moyennes comparées selon le test de Newman-Keuls au seuil de $5 \%$. 


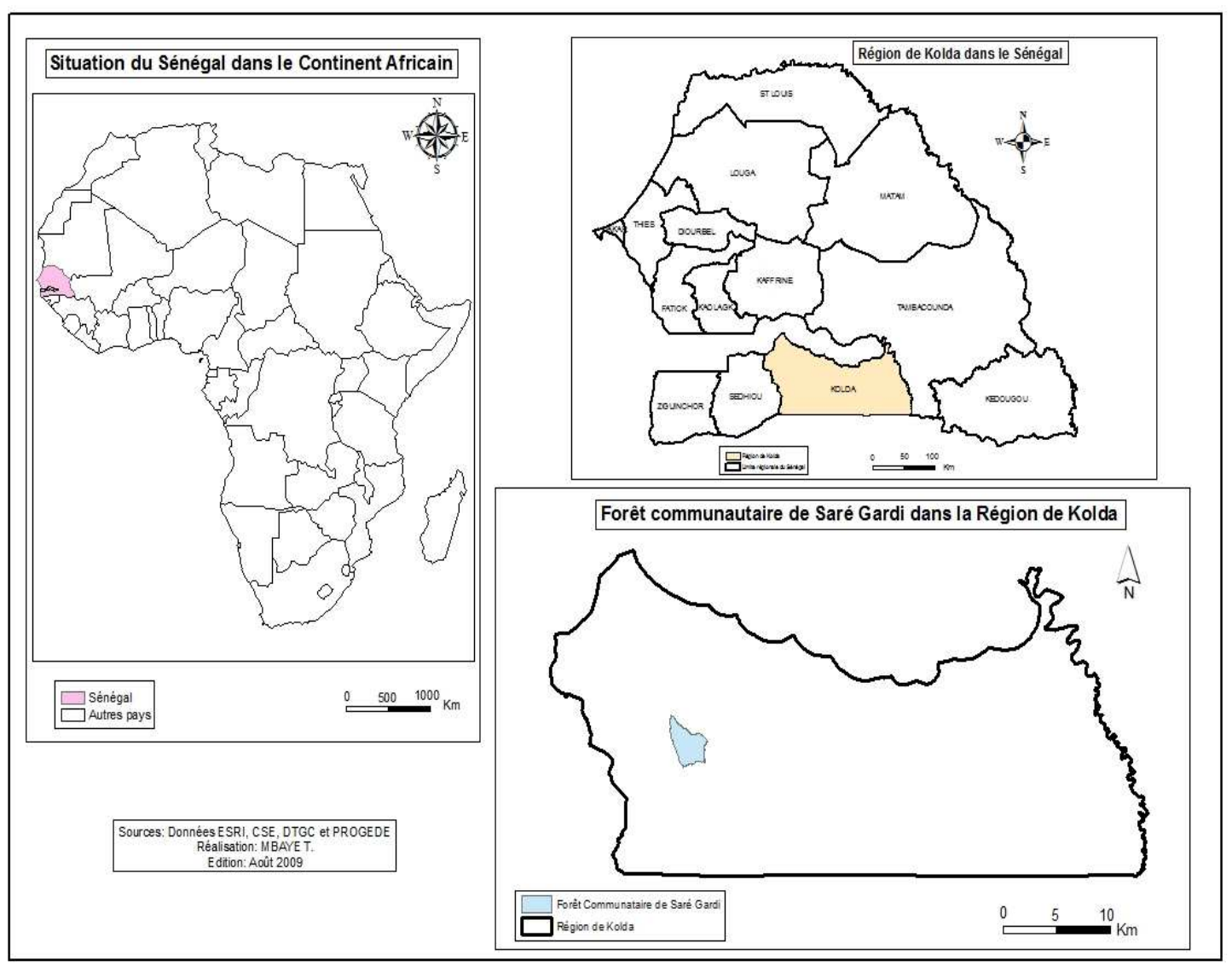

Figure 1: Localisation du site d'étude.

\section{RESULTATS}

Fréquence des classes de diamètre de coupe dans les différentes zones

Dans la ZA, la classe de diamètre [3,6[ $\mathrm{cm}$ est celle où il $\mathrm{y}$ a le plus grand nombre d'individus coupée avec $47,72 \%$ contrairement à la ZNA où la classe de diamètre $[0,3[\mathrm{~cm}$ domine avec $58,16 \%$. (Tableau1).

En dehors de la classe de diamètre $[0,3[$ $\mathrm{cm}$ dans la ZA avec $34,93 \%$, les autres ne présentent pas de différences significatives. Néanmoins, toutes les classes de diamètres, dans les deux zones ont été coupées.

Fréquence des classes hauteur de coupe dans les différentes zones

Dans la ZA, les résultats montrent que les coupes au ras du sol tant dans la ZA que dans la ZNA sont les plus fréquentes avec respectivement $80,36 \%$ et $69,74 \%$ (Tableau 2). Pour les autres classes de hauteur de coupe, il n'y a pas de différences significatives. Néanmoins, les coupes ont concerné toutes les classes de hauteur dans les deux zones.

\section{Fréquence des classes de hauteur de rejets dans les différentes zones}

Les analyses de variance effectuées sur les données de fréquence des classes de hauteur de rejet révèlent des différences significatives dans la zone aménagée et dans la zone non aménagée (Tableau 3).

Dans chacune des deux zones, les classes de hauteur moyenne et basse des rejets ont des fréquences qui sont significativement plus importantes que celles des classes de 
hauteur haute. Néanmoins, dans la ZNA la classe de hauteur de rejet haute est représentée à $2,20 \%$.

\section{Fréquence des classes de nombre tiges dans les différentes zones}

Le Tableau 4 montre que les classes de nombre de tiges dans la ZA et la ZNA comprises entre $[0,10[$ et $[10,20[$ sont les plus représentées avec respectivement $65,83 \%$ et $92,76 \%$ dans la première classe et $27,21 \%$ et $6,30 \%$ dans la seconde.

Pour les autres classes de nombre de tiges, il n'y a pas de différence significative. Les classes de nombre de tiges sont plus présentes dans la zone aménagée avec les classes $[0,10[,[10,20[,[20,30[,[30,40[$ et [40,50[ contrairement à la zone non aménagée où seules les classes $[0,10[,[10,20[,[20,30[$ sont concernées.

\section{DISCUSSION}

Nos résultats ont montré que les classes de diamètre de coupe $[0,3[\mathrm{~cm}$ et $[3,6[$ $\mathrm{cm}$ dans la ZA comme dans la ZNA sont les plus coupées. Ces résultats confirment ceux de Peltier (2012) qui montre que plus de 50\% des coupes n'ont pas atteint $10 \mathrm{~cm}$ de diamètre comme le stipule le plan d'aménagement. De même, pour Manga (2008) plus le diamètre d'exploitabilité augmente, moins les populations sont enclines à les exploiter. Néanmoins, d'après le plan d'aménagement, toutes les coupes devaient concerner les diamètres compris entre 10 et $25 \mathrm{~cm}$, ce qui est contraires à nos résultats.

En effet, les populations locales, ne voulant pas exploiter le charbon, ont soit coupé sans tenir compte des prescriptions techniques de l'aménagement, soit contractualisé avec les exploitants traditionnels des ZNA.

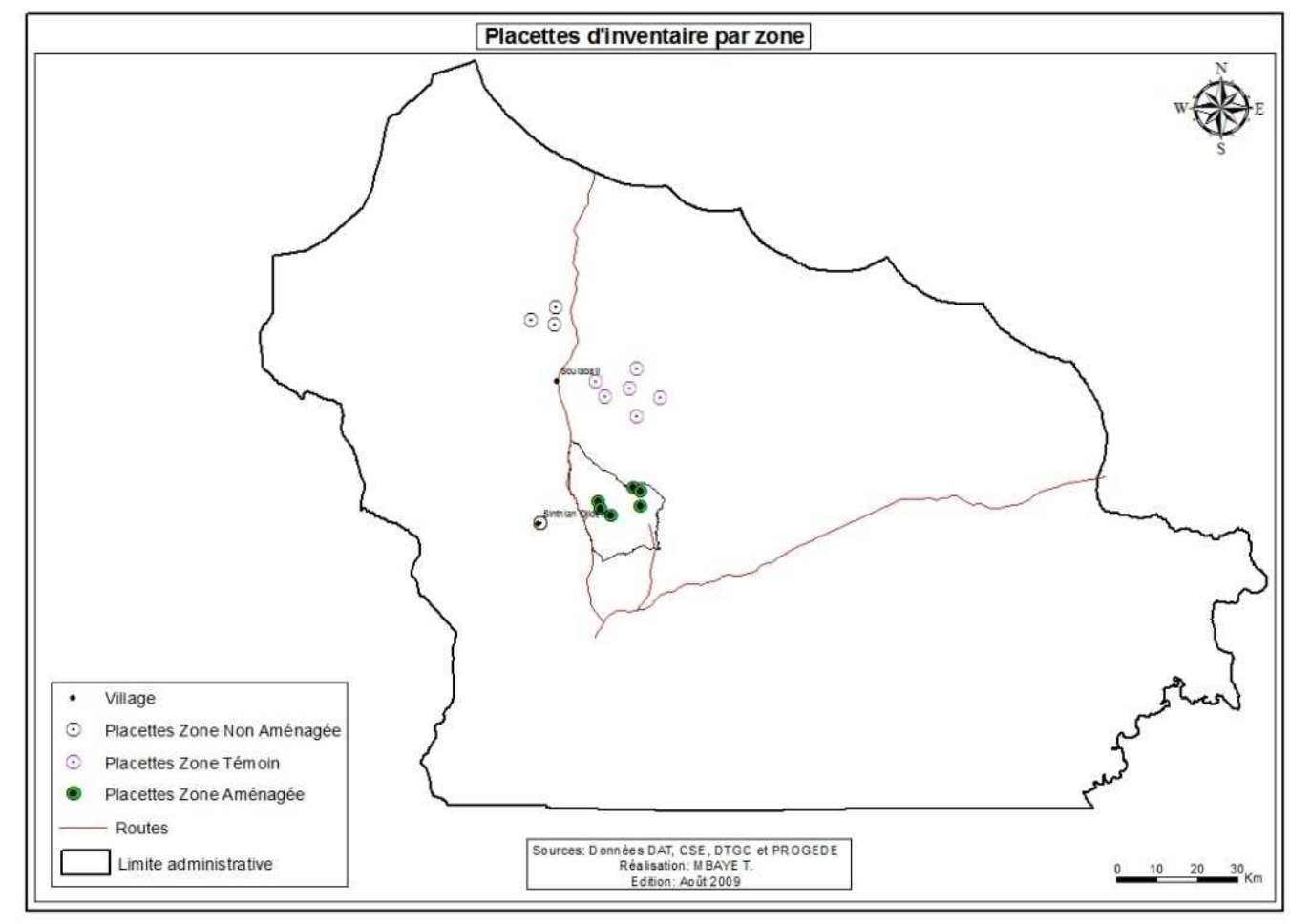

Figure 2 : Localisation des placettes d'inventaire. 
Tableau 1 : Fréquence relative des espèces coupées dans la ZA et la ZNA.

\begin{tabular}{|c|c|c|}
\hline \multirow{2}{*}{ Espèces } & \multicolumn{2}{|c|}{ Fréquence relative (\%) } \\
\hline & Zone non aménagée & Zone aménagée \\
\hline Acacia macrostachia & $1,05^{\mathrm{b}}$ & \\
\hline Afrormosia laxiflora & $0,12^{\mathrm{b}}$ & \\
\hline Anona senegalensis & $1,73^{\mathrm{b}}$ & \\
\hline Bombax costatum & $10,33^{\mathrm{b}}$ & \\
\hline Combretum glutinosum & $55,02^{\mathrm{a}}$ & $99,20^{\mathrm{a}}$ \\
\hline Combretum micranthum & $3,66^{\mathrm{b}}$ & \\
\hline Combretum nigricans & & $0,80^{\mathrm{b}}$ \\
\hline Combretum sp & $1,57^{\mathrm{b}}$ & \\
\hline Cordyla pinnata & $2,47^{\mathrm{b}}$ & \\
\hline Detarium microcarpum & $0,12^{\mathrm{b}}$ & \\
\hline Erythrina senegalensis & $0,14^{\mathrm{b}}$ & \\
\hline Exalobus menopotalis & $1,13^{\mathrm{b}}$ & \\
\hline Gardenia termifolia & $0,28^{\mathrm{b}}$ & \\
\hline Lannea acida & $1,00^{\mathrm{b}}$ & \\
\hline Lannea voluptina & $0,26^{\mathrm{b}}$ & \\
\hline Ostryosderris stuhlmannii & $2,86^{\mathrm{b}}$ & \\
\hline Piliostigma thonninguii & $0,28^{\mathrm{b}}$ & \\
\hline Prosopis africana & $0,27^{\mathrm{b}}$ & \\
\hline Pterocarpus erinaceus & $2,45^{\mathrm{b}}$ & \\
\hline Pterocarpus lucens & $0,79^{\mathrm{b}}$ & \\
\hline Sterculia setugera & $0,17^{\mathrm{b}}$ & \\
\hline Stéreospermum kuntianum & $2,09^{\mathrm{b}}$ & \\
\hline Strychnos spinosa & $1,58^{\mathrm{b}}$ & \\
\hline Terminalia avicennoides & $0,98^{\mathrm{b}}$ & \\
\hline Terminalia macroptera & $7,83^{\mathrm{b}}$ & \\
\hline Vitex donania & $1,79^{\mathrm{b}}$ & \\
\hline Valeur de $\mathrm{p}$ & $<0,0001$ & $<0,0001$ \\
\hline
\end{tabular}

Tableau 2 : Evolution des fréquences des classes de diamètres de coupe dans les différentes zones $(\%)$.

\begin{tabular}{lcc}
\hline \multirow{2}{*}{\begin{tabular}{l} 
Classe diamètre de coupe \\
\cline { 2 - 3 }
\end{tabular}} & \multicolumn{2}{c}{ Fréquence $(\%)$} \\
\hline$[0,3[$ & $34,93^{\mathrm{b}}$ & Zone non aménagée \\
{$[3,6[$} & $47,72^{\mathrm{c}}$ & $58,16^{\mathrm{b}}$ \\
{$[6,9[$} & $10,13^{\mathrm{a}}$ & $12,23^{\mathrm{a}}$ \\
{$[9,12[$} & $3,26^{\mathrm{a}}$ & $3,75^{\mathrm{a}}$ \\
{$[12,15[$} & $2,13^{\mathrm{a}}$ & $8,00^{\mathrm{a}}$ \\
15 et + & $1,83^{\mathrm{a}}$ & $6,60^{\mathrm{a}}$ \\
\hline valeur de $\mathrm{p}$ & $<0,0001$ & $11,26^{\mathrm{a}}$ \\
\hline Les valeurs d'une même colonne suivies de mêmes lettres ne sont pas significativement différentes au seuil de $5 \%$ \\
\multicolumn{2}{l}{ (Newman Keuls). }
\end{tabular}


Tableau 3 : Evolution des fréquences des classes de hauteur de coupe dans les différentes zones $(\%)$.

\begin{tabular}{lcc}
\hline $\begin{array}{l}\text { Classe de Hauteur } \\
\text { de coupe } \mathbf{( c m )}\end{array}$ & \multicolumn{2}{c}{ Fréquences des classes de hauteur de coupe (\%) } \\
\cline { 2 - 3 } Ras du sol & Zone aménagée & Zone non aménagée \\
{$[1,10[$} & $80,36^{\mathrm{b}}$ & $69,74^{\mathrm{b}}$ \\
{$[10,20[$} & $5,49^{\mathrm{a}}$ & $1,52^{\mathrm{a}}$ \\
{$[20,30[$} & $5,09^{\mathrm{a}}$ & $9,45^{\mathrm{a}}$ \\
30 et + & $4,93^{\mathrm{a}}$ & $5,24^{\mathrm{a}}$ \\
\hline Valeur de $\mathrm{p}$ & $4,13^{\mathrm{a}}$ & $14,05^{\mathrm{a}}$ \\
\hline Les valeurs d'une même colonne suivies de mêmes lettres ne sont pas significativement différentes au seuil de $5 \%$ \\
\multicolumn{2}{l}{ (Newman Keuls). }
\end{tabular}

Tableau 4 : Evolution des fréquences des classes de nombre de tiges dans les différentes zones $(\%)$.

\begin{tabular}{lcc}
\hline \multirow{2}{*}{ Classe nombre de tiges } & \multicolumn{2}{c}{ Fréquence (\%) } \\
\cline { 2 - 3 } & Zone aménagée & Zone non aménagée \\
\hline$[0,10[$ & $65,83 \mathrm{c}$ & $92,76 \mathrm{c}$ \\
{$[10,20[$} & $27,21 \mathrm{~b}$ & $6,30 \mathrm{~b}$ \\
{$[20,30[$} & $4,85 \mathrm{a}$ & $0,93 \mathrm{a}$ \\
{$[30,40[$} & $1,81 \mathrm{a}$ & $0,00 \mathrm{a}$ \\
{$[40,50[$} & $0,30 \mathrm{a}$ & $0,00 \mathrm{a}$ \\
{$[50,60[$} & $0,00 \mathrm{a}$ & $0,00 \mathrm{a}$ \\
{$[60,70[$} & $0,00 \mathrm{a}$ & $0,00 \mathrm{a}$ \\
{$[70,80[$} & $0,00 \mathrm{a}$ & $0,00 \mathrm{a}$ \\
{$[80,90[$} & $0,00 \mathrm{a}$ & $0,00 \mathrm{a}$ \\
{$[90,100[$} & $0,00 \mathrm{a}$ & $0,00 \mathrm{a}$ \\
100 et + & $0,00 \mathrm{a}$ & $0,00 \mathrm{a}$ \\
\hline Valeur de $\mathrm{p}$ & $<0,0001$ & $<0,0001$ \\
\hline Les valeurs d'une même colonne suivies de mêmes lettres ne sont pas significativement différentes au seuil de 5\%
\end{tabular}

Ces derniers ont transposé leurs habitudes d'exploitation sans restriction aucune. Ces résultats confirment ceux de Manga (2008) pour qui l'explication serait liée au fait que beaucoup d'exploitants préfèrent couper les petites tiges pour diminuer le temps de fabrication du charbon. Toutefois, pour Nouvellet (1992), il faut un taillis simple, un diamètre minimum d'exploitabilité (DME) compris entre 8 et 15 $\mathrm{cm}$ avec une courte rotation (7 ans \pm 2 ans). Peltier et al. (1994) proposent quant à eux deux diamètres minimum d'exploitabilité suivant les espèces concernées. Pour Guiera senegalensis et Combretum micranthum, le DME à la base est de $6 \mathrm{~cm}$ et de $8 \mathrm{~cm}$ à la base pour Combretum nigricans et Combretum glutinosum. Ces deux espèces sont les seules destinées à la coupe dans la ZA.

Pour la hauteur de coupe, nos résultats montrent que les coupes au ras du sol dominent dans la ZA avec $80,36 \%$, comme indiqué dans le plan d'aménagement, et dans la ZNA avec $69,74 \%$. Ces résultats confirment les travaux de Ouedraogo (2004) et de Savadogo (2007) en zone aménagée.

Dans la ZNA, nos résultats indiquent que les coupes non rases représentent 30,26\%. Ces résultats confirment Manga (2008) qui signale que c'est pour disposer de plus de cépées et à des hauteurs atteignant $1 \mathrm{~m}$ pour des raisons pratriques dans la coupe de bois 
des taillis (moins fatiguant pour les populations). C'est le cas également au Niger où Larwanou et Saadou (2004) indiquent que les coupes en têtard effectuées à environ 1,5 et $2 \mathrm{~m}$ assure une meilleure croissance et où Robert et Moussa (2003) montrent que ces rejets sont à l'abri des animaux.

Dans la ZA, même si les coupes devaient être rases d'après le plan d'aménagement, les coupes non rases peuvent être pertinentes comme le suggère Bellefontaine (1995a) grâce au développement de brins vivaces et de bourgeons proventifs comme le montre Kairé (2000). D'autant que Peltier (2012), après avoir suivi l'évolution de massifs aménagés dans la même zone, a montré que presque tous les arbres coupés survivent et donnent un taillis. Les rejets situés à la base de la souche sont plus vigoureux que ceux en haut, souvent détachés par le vent, le bétail ou les hommes qui manient le bois.

\section{Conclusion}

Il ressort de notre étude que les petits diamètres de coupe, compris entre $[0,3[\mathrm{~cm}$ et [3,6[ cm sont les plus exploités et, généralement, en coupes rases. Néanmoins, beaucoup de coupes non rases ont été notées, surtout en ZA qui témoigne d'un non respect des prescriptions techniques du plan d'aménagement qui recommandait des diamètres de coupe compris entre 10 et $25 \mathrm{~cm}$ et des coupes rases. Le choix des espèces est aussi déterminant pour un aménagement, mais il ne faudrait pas le circonscrire à un nombre assez limité pour ne pas favoriser un déséquilibre.

\section{REFERENCES}

Bailly C, Barbier C, Clement J, Goudet JP, Hamel O. 1982. Les problèmes de la patisfaction des besoins en bois en Afrique tropicale sèche:Connaissances et Incertitudes. Rev. BFT, 197: 23-43.

Bellefontaine R. 1997. Aménagement des forêts naturelles des zones tropicales sèches, FAO, 307p.
Bellefontaine R. 1995a. Choix du type de régénération pour aménager les forêts tropicales sèches. Cinquième réunion tripartite, Korhogo, 21-23 mars 1995, IDEFOR, Abidjan, 5 p.

Bellefontaine R. 1995b. Synthèse des espèces des domaines sahélien et soudanien qui se multiplient naturellement par voie végétative. Actes du séminaire sur les Ecosystèmes Sahéliens Contractés. Niamey, 20-24 novembre 1995, Springler Verlag.

Björkdahl G, Camara AA. 2003. Régénération, croissance et productivité de Combretum glutinosum après exploitation de peuplements naturels au Sénégal oriental, pp. 93101. In Aménagement intégré des forêts naturelles des zones tropicales sèches de l'Afrique de l'Ouest, Ouagadougou, 16-20 nov 1998. CNRST Ouagadougou, 309 p.

Boye A. 2000. L'Etude Prospective $d u$ Secteur Forestier en Afrique (FOSA). Centre de Suivi Ecologique. République du Sénégal, Dakar.

Diagne M. 2001. Vulnérabilité des productions agricoles aux changements climatiques au Sénégal. MJEHP / Direction de l'environnement / Programme d'Assistance des Pays Bas sur les changements climatiques. Dakar, ministère de l'environnement, $36 \mathrm{p}$.

Faye M, Akakpo K, Dramani LGA. 2003. Deuxième enquête sur les flux de combustibles ligneux au Sénégal ; Rapport de fin de mission; PROGEDE ; 59 p.

Faye E, Diallo H, Samba SAN, Touré MA, Dramé A, Fall B, Lejoly J, Diatta M, Kaïré M, De Cannière C, Mahy G, Bogaert J. 2013. Importance de la méthode de coupe sur la régénération de Combretaceae du Bassin arachidier sénégalais. Tropicultura, 31(1): 44-52. 
Kabore C, Ouedraogo K. 1995. Aménagement des forêts des zones sèches: le cas du Burkina Faso. Rapport de consultants FAO, Rome, 56 p.

Kaire M. 2000. La production ligneuse des jachères et son utilisation par l'homme du Sénégal, thèse $3^{\text {ème }}$ cycle, Marseille (coencadrement), 113 pages + annexes.

Larwanou M, Saadou M. 2004. Influence du régime de coupe sur la régénération de l'espèce Acacia nilotica (L.) Wild. dans une formation de bas-fonds (Forêt de Korop) au Niger, bulletin de recherche agronomique du Bénin, $\mathrm{N}^{\circ} 46$ décembre 2004, 8 p.

Manga A. 2008. L'arbre, le chantier, la meule: Glissement vers la fin d'une logique de prélèvement «pérenne». Analyse et cartographie de la production du charbon de bois dans le département de Tambacounda (Sénégal), Doctorat troisième cycle, Département de géographie, FLSH, UCAD, Dakar, 283 p.

Nouvellet Y. 1992. Evolution d'un taillis de formation naturelle en zone soudanienne du Burkina Faso. Thèse Université Pierre et Marie Curie, Paris VI, 356p.

Peltier R, Lawali ELHM, Montagne P. 1994. Aménagement villageois des brousses tachetées au Niger. $1^{\text {ère }}$ parie le milieu : potentiel et contraintes. Bois et Forêts des Tropiques, 242 : 59-76.

Peltier R. 2012. Evaluation de la mise en œuvre des plans d'aménagement forestier dans les forêts communautaires appuyées par le programme USAID Wula Nafaa au Sénégal, $60 \mathrm{p}$.

Ribot JC. 1999. Un historique de peur: Les pronostics de déforestation des forêts des zones sèches de l'Afrique occidentale. Harvard University, Glob. Ecol. And Biogeo. Let., 15 p.

Robert N, Moussa MD, 2003. Contribution à l'étude de la régénération de Combretum nigricans dans les zones de Bellaré et Kirtachi. Rapport, Projet énergie domestique, Ministère de l'Hydraulique et de l'environnement, Niamey, Niger, 25 p.

Sawadogo A. 1991. L'arbre et la forêt dans l'aménagement du territoire: aménagement intégré des espaces ruraux. Actes du 10ème Congrès Forestier Mondial, Paris. Rev. For. Fran., 3: 9-12.

Sawadogo A. 2002. Influence of selective tree cutting, livestock and prescribed fire on herbaceous biomass in the Savannah woodland of Burkina Faso, West Africa. Rev. Agric., Ecos. Env., 105: 335-345.

Sow B, Mbaye M, Ickowcz A, Rippstein G, Lesueur D. 2002. Rôle et implication des comités villageois et de développement dans le fonctionnement d'un programme de gestion participative des terroirs agro-sylvopastoraux : exemple du PROGEDE au Sénégal, Tambacounda, $10 \mathrm{p}$.

Yossi H. 1996. Dynamique de la végétation post-culturale (strate ligneuse) au Mali. Thèse de 3 e cycle, ISFRA de Bamako, Mali, $141 \mathrm{p}$. 